\title{
The star cluster population of M 51
}

\section{Cluster disruption and formation history}

\author{
M. Gieles ${ }^{1}$, N. Bastian ${ }^{1}$, H. J. G. L. M. Lamers ${ }^{1,2}$, and J. N. Mout ${ }^{1}$ \\ 1 Astronomical Institute, Utrecht University, Princetonplein 5, 3584 CC Utrecht, The Netherlands \\ e-mail: gieles@astro.uu.nl \\ 2 SRON Laboratory for Space Research, Sorbonnelaan 2, 3584 CA Utrecht, The Netherlands
}

Received 21 February 2005 / Accepted 27 May 2005

\section{ABSTRACT}

In this work we concentrate on the evolution of the cluster population of the interacting galaxy M 51 (NGC 5194), more precisely the timescale of cluster disruption and possible variations in the cluster formation rate. We present a method to compare observed age vs. mass number density diagrams with predicted populations including various physical input parameters like the cluster initial mass function, cluster disruption, cluster formation rate and star bursts. If we assume that the cluster formation rate increases at the moments of the encounters with NGC 5195, we find an increase in the cluster formation rate of a factor of $3.0_{-1.2}^{+4.6}$, combined with a disruption timescale which is slightly higher than when assuming a constant formation rate $\left(t_{4}=2.0_{-1.1}^{+2.3} \times 10^{8} \mathrm{yr}\right.$ vs. $\left.1.0_{-0.5}^{+0.6} \times 10^{8} \mathrm{yr}\right)$. The measured cluster disruption time is a factor of 5 shorter than expected on theoretical grounds. This implies that the disk of M 51 is not a preferred location for survival of young globular clusters, since even clusters with masses on the order of $10^{6} M_{\odot}$ will be destroyed within a few Gyr.

Key words. galaxies: spiral - galaxies: individual: M 51 - galaxies: star clusters

\section{Introduction}

The goal of this series of papers is to understand the properties of the entire star cluster population of the interacting spiral galaxy M 51. These properties include the age and mass distribution of the cluster population. Additional properties are the survival rate of the clusters, as well as any relations between the observed properties. These relations may be used to constrain cluster formation and destruction scenarios.

In order to study the above properties, we exploit the large amount of HST broad-band archival data on M 51, which covers roughly $50 \%$ of the observed surface area of M 51, and covers a broad spectral range ( $U V$ to $N I R$ ). The large spatial coverage is necessary in order to obtain a large sample of clusters for carrying out a statistical analysis, and the broad spectral range allows accurate determination of the individual cluster properties (Bik et al. 2003; Anders et al. 2004). A preliminary analysis of a subset of the M51 cluster population was carried out by Bik et al. (2003, hereafter Paper I), who introduced the method used to determine the cluster properties and derived the age and mass distributions of the cluster sample roughly $2 \mathrm{kpc}$ to the north-east of the nucleus.

Bastian et al. (2005, hereafter Paper II) extended the survey to include the entire inner $\sim 5 \mathrm{kpc}$ of M 51 , and found 1152 clusters, 305 of which had accurate size determinations. In that work we extended the age distribution analysis of Paper I and found evidence for a cluster formation rate increase $\sim 50-70 \mathrm{Myr}$ ago. This corresponds to the last close passage of NGC 5195 and M 51 (Salo \& Laurikainen 2000). Additionally we found that $68 \pm 15 \%$ of the clusters forming in M 51 will disrupt within the first $\sim 10$ Myr after their formation, independent of their mass, so-called infant mortality. For the resolved cluster sample, we found that the size distribution (the number of clusters as a function of their effective radius) can be well fit by a power-law: $N \mathrm{~d} r_{\text {eff }} \propto r_{\text {eff }}{ }^{-\eta} \mathrm{d} r_{\text {eff }}$, with $\eta=2.2 \pm 0.2$, which is very similar to that found for Galactic globular clusters. Finally, we did not find any relation between the age and mass, mass and size, or distance from the galactic center and cluster size.

In this study we focus on the evolution of the population of clusters in M 51, in particular the timescale of cluster disruption and possible variations in the cluster formation rate. Cluster disruption of multi-aged populations, which excludes the galactic globular clusters, has been the subject of many earlier studies: e.g. Hodge (1987) for the SMC and Battinelli \& Capuzzo-Dolcetta (1991) for the Milky Way. In this study we will take mass dependent disruption into account, since the time needed to destroy half of the cluster population, which has been estimated in earlier work, will strongly depend on the mean mass of the sample and the lower mass limit of the sample. In addition, we here want to study the effect of variations in the formation rate, which is usually kept constant. 
Boutloukos \& Lamers (2003) have developed a method to derive the disruption timescale based on the age and mass distributions of a magnitude-limited cluster sample. They found that the disruption time of clusters in M51 is a factor of 15 shorter than the one for open clusters in the solar neighborhood. Lamers et al. (2005a) showed that part of the difference can be explained by the difference in density of the cluster environment and that the disruption time of clusters depends on the clusters initial mass and the galaxy density as $t_{\text {dis }} \propto M_{\mathrm{i}}^{0.62} \rho_{\text {gal }}^{-0.5}$, based on the results of $N$-body simulations. The disruption time of clusters in M 51 was still about a factor of 10 lower than the predicted value. In this work we are particularly interested in seeing if a short disruption timescale can be mimicked by an increasing cluster formation rate, and how the assumed disruption law influences the derived timescales. To this end we generated artificial cluster samples with parameterized global characteristics (e.g. time-dependent cluster formation rates, disruption laws, infant mortality rates, and mass functions). We then compare these models with the derived age and mass distributions of the cluster population of M51 to derive the best fit parameters for the population as a whole.

The structure of the paper is as follows: in Sect. 2 the observations of the cluster population of M 51 are presented. In Sect. 3 we investigate to what extent the disruption time depends on different cluster and galaxy parameters. Section 4 describes the steps we will take in our models, where the details of the models we used to generate artificial cluster populations will be explained in Sect. 5. The results of the fits are given in Sect. 6. A discussion of the implication of the results is given in Sect. 7, and the conclusions are presented in Sect. 8.

\section{The observations}

\subsection{Fitting the observed spectral energy distribution}

From archival HST broadband photometry we have derived the age, mass, and extinction of 1152 clusters in M51 (Paper II), using the three-dimensional maximum likelihood fitting (3DEF) method. Details about the 3DEF method can be found in Paper I. In summary, the spectral energy distribution of each cluster is compared with cluster evolution models. In this case the GALEV simple stellar population (SSP) models (Anders et al. 2003; Schulz et al. 2002) for solar metallicity and Salpeter IMF are used. For each age a series of different extinctions is then applied to the models and all combinations of age and extinction are compared to the data. The lowest $\chi^{2}$ is kept, and from the absolute magnitude at that age, the mass is determined. Detailed tests of the accuracy and reliability of the derived parameters are presented in Paper II. In the present work, we further investigated the accuracy of our fitting method and used the data set to develop a model that describes the global properties of the cluster system.

\subsection{The age and mass distribution of clusters}

The ages and present masses of the 1152 clusters are plotted in the top panel of Fig. 1. In order to be able to compare our observations with simulated cluster samples, we binned the data in
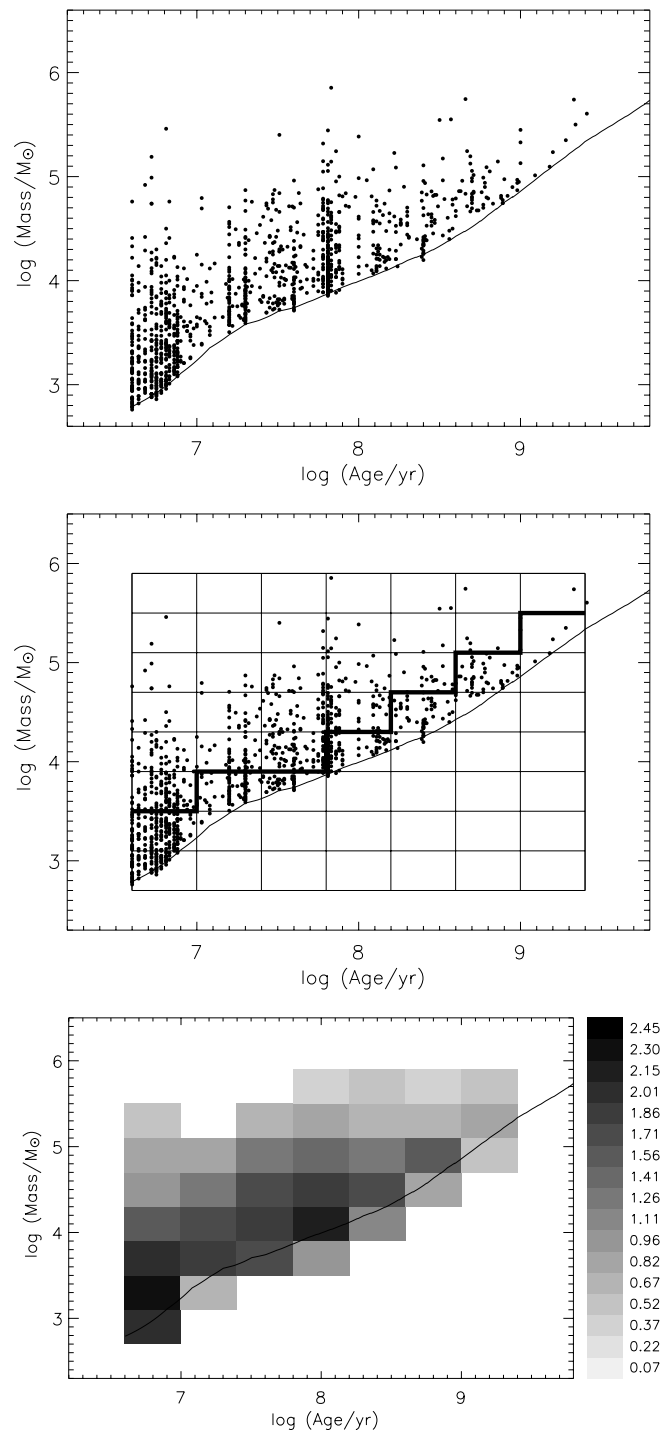

Fig. 1. Ages and present masses of the 1152 clusters identified in Paper II. Top: original data, where every point represents a cluster. Middle: same data as in the top panel overplotted with the grid used to bin the data. Above the dark line are the bins not affected by the detection limit. Bottom: logarithmic density plot of the same sample, where dark regions represent more clusters. The right hand scale shows how the grey values correspond to the logarithm of number. The line in all three plots is the $90 \%$ completeness limit $(F 439 \mathrm{~W}=22.6 \mathrm{mag})$.

logarithmic number density plots of the age vs. mass distribution. Clusters were counted in bins of 0.4 age dex by 0.4 mass dex (Fig. 1, middle), and the result illustrated in the bottom panel of Fig. 1. A few striking features can be learned from this diagram:

1. a burst in the cluster formation rate (CFR) between 50 and $70 \mathrm{Myr}$, corresponding to the most recent interaction with the companion galaxy NGC 5195;

2. a short-lived young population with ages $<10 \mathrm{Myr}$. In Paper II we found that $\pm 68 \%$ of these young clusters will dissolve within $10 \mathrm{Myr}$, independent of their mass;

3. evolutionary fading under the detection limit, which makes it harder to detect old low-mass clusters. The increasing 
line in Fig. 1 shows how the $90 \%$ completeness limit in the $F 439 \mathrm{~W}$ band (22.6 mag) corresponds to different masses at different ages;

4. an apparent increase in the mass of the most massive cluster with age. This is a binning effect: the older age bins span more time and therefore contain more clusters, and the chance of finding a more massive cluster at older ages is higher due to the size of sample effect (Hunter et al. 2003). We tried to use the method of Hunter et al. (2003) to derive the cluster formation rate, but found that the increase in the maximum mass is much too shallow. This probably means that M51 has reached the maximum cluster mass $\left(\sim 10^{6} M_{\odot}\right)$ when the relation of Hunter et al. (2003) does not apply anymore, since the maximum cluster mass found at a certain age is then no longer determined anymore by sampling statistics. This is the topic of a subsequent study (Gieles et al. 2005).

We found that a large fraction $( \pm 68 \%)$ of the clusters younger than $10^{7} \mathrm{yr}$ dissolves independent of mass, probably due to the removal of the primordial gas (e.g. Kroupa 2004; Geyer \& Burkert 2001; Lada \& Lada 2003). After these critical $10^{7} \mathrm{yr}$, the surviving clusters will dissolve due to the tidal field of the host galaxy and external perturbations from, for example, encounters with giant molecular clouds (GMCs). The disruption of clusters is in that sense a two step process. Here we will use the resulting age/mass distribution to study the disruption of clusters with ages larger than $10^{7} \mathrm{yr}$, i.e. the second step in the disruption process.

\subsection{Artifacts introduced by the age-fitting method}

We want to see whether the 3DEF method (Sect. 2.1), used to derive ages, masses, and extinctions from the photometry, introduces systematic artifacts. More important, could it affect our results of the disruption time or formation rate? For instance, are there systematically old clusters fitted with young ages or the other way around?

The uncertainty in the derived ages, extinctions, and masses from broad-band photometry is caused mainly by two effects:

1. differences between the real integrated colors of the clusters and the models, for example due to stochastic sampling of the stellar IMF, which cannot be taken into account in the SSP models, or errors in the stellar isochrones used;

2. systematic errors introduced by the age-fitting method and the applied selection effects.

Of course, the first effect cannot be corrected for, unless we are able to compare photometric determined ages directly to spectroscopically determined ages, which is unfortunately not feasible for a whole population of clusters. In addition, although spectroscopically derived ages are more accurate, they are hampered by their own problems (Brodie et al. 1998). In addition, the age derivation will depend on the choice of the adopted SSP models. Variations in the metallicity and the IMF will affect the derived ages. A detailed comparison of the data with models of different metallicity was carried out in
Paper II. An earlier study of the age distribution of M 51 used Starburst99 models (Bastian \& Lamers 2003), and they found a very similar age distribution.

The second effect can be quantified with the use of artificial cluster populations. Earlier studies (e.g. Anders et al. 2004; de Grijs et al. 2005) have already shown the importance of using a long wavelength baseline ( $U$ to NIR) to age-date young clusters. Here we make an attempt to quantify possible systematic errors introduced by the age-fitting method and see whether we can correct for them or not.

To quantify the artifacts introduced by the fitting routine, an artificial cluster sample including simulated observational errors and extinction values was generated and fitted with the same fitting procedure as used for the data (Sect. 2.1). We started with a sample of clusters equally spread in $\log (\mathrm{Age} / \mathrm{yr})$ and $\log \left(M / M_{\odot}\right)$ space. In total 201 time steps between $\log ($ Age $/ \mathrm{yr})=6$ and $\log ($ Age $/ \mathrm{yr})=10$ and 161 masssteps between $\log \left(M / M_{\odot}\right)=2$ and $\log \left(M / M_{\odot}\right)=7$ were generated. The $G A L E V$ models have $\log (\mathrm{Age} / \mathrm{yr})=6.6$ as youngest model, so clusters with younger ages were given that age. The magnitudes as a function of age and mass were taken from the GALEV SSP models. Observational uncertainties were applied as a function of magnitude as was done in Paper II; the observed errors in the magnitudes of clusters in M 51 can be well approximated by $\Delta \operatorname{mag}_{\lambda}=10^{d_{1}+d_{2} \times \operatorname{mag}_{\lambda}}$. The values for $d_{1}$ and $d_{2}$ for the different filters are results from analytical functions fitted to the observed errors and magnitudes and are given in Table 4 of Paper II. Ideally, we then apply the same extinction to the model clusters as the M 51 clusters have. Unfortunately, the only information we had was the extinction we measured, which of course could already be polluted with artifacts. To get an estimate of the uncertainty in the measured extinction, we started with a sample of clusters with no extinction applied. When we fit this population with the 3DEF method, we found that $20 \%$ of the sources was fitted with some extinction. This is quite a large number, but fortunately $90 \%$ of these sources have extinction values lower than $E(B-V)=0.1 \mathrm{mag}$ The maximum $E(B-V)$ found is $1 \mathrm{mag}$.

The next step is to apply an extinction model close to what we observe. To this end $E(B-V)$ extinction values were chosen randomly from a Gaussian distribution centered at 0 with $\sigma=0.10$ for clusters younger than $\log ($ Age $/$ yr $)=7.3$ and $\sigma=0.05$ for clusters older than $\log (\mathrm{Age} / \mathrm{yr})=7.3$. The values for $\sigma$ agree with the value we found for the mean extinction in Paper II. There we found that these values are the average extinction for these two age groups. The higher extinction for young ages is caused by the presence of the left-over dust around the cluster. Negative extinctions were set to 0 , resembling the extinction distribution of the data where half of the clusters had $E(B-V)=0$ (See Fig. 8 of Paper II). An age-dependent maximum extinction was applied of the form: $E(B-V)_{\max }(t)=5-0.5 * \log (t)$. This is a little bit lower than the observed maximum extinction, but we know that some of the observed high values could be caused by wrong fits. This still resembles the observed extinction behavior quite well. The resulting magnitudes were than cut off at our completeness limits in each filter. In this way we created the spectral energy distributions of a large artificial cluster sample with age, mass, and 


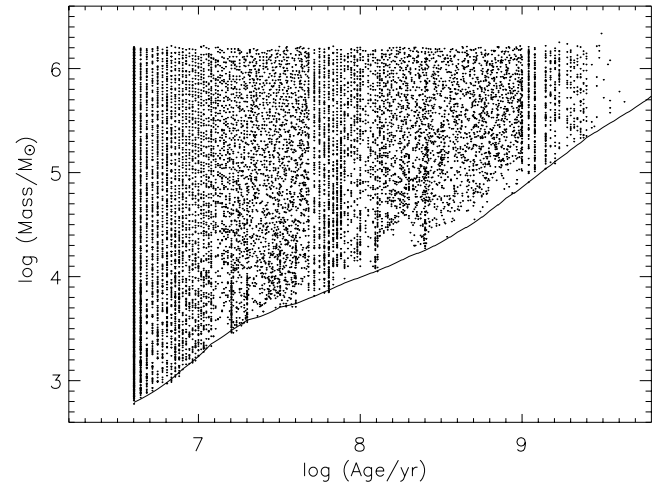

Fig. 2. Age/mass diagram of artificial sample after fitting with the method described in Sect. 2.1. The deviations from equally spaced dots in $\log (\mathrm{Age} / \mathrm{yr}) / \log \left(M / M_{\odot}\right)$ are caused by the applied observational errors and the fitting routine.

extinction known for each cluster. These were fitted with the 3DEF method (Sect. 2.1).

The result of the fitted simulation is shown in Fig. 2. A direct comparison with the observed age-mass diagram of M51 clusters (Fig. 1, top) shows that there are features present in the data which are not visible in the fitted simulation. For example, there is a gap at $6.9<\log ($ Age $/ y r)<7.1$ in the M 51 cluster sample, which seems to appear at slightly higher ages in the fitted simulations $(7.1<\log ($ Age $/ y r)<7.2)$. This suggests that the artifacts in the data are caused not only by our applied selection effects or our age-fitting technique. In the top left panel of Fig. 3 we show the fitted age versus the input age for the simulated cluster sample. A large number of clusters with wrong ages are fitted with a $\log ($ Age $/ y r) \simeq 7$. We found for $87 \%$ of the modeled clusters that the fitted age was the same as the input age within 0.4 dex (Fig. 3, top right). For the mass, $97 \%$ was fitted correctly within 0.4 dex (Fig. 3, bottom right) and $92 \%$ of the extinction values were fitted back within 0.05 mag (Fig. 3, bottom left). We have to realize that the strength of the artifacts depends on the number of input clusters at each age and mass bin. We have not attempted to match the observations in this stage, since we are only interested in relative errors. For example, the horizontal spur at $\log ($ Age/yr) $\simeq 7$ in Fig. 3 (top, left) is populated with clusters with input ages up till a few times $10^{9} \mathrm{yr}$. The number of clusters with that age in our M 51 sample is very low (see Fig. 1, top).

\subsection{Correcting the data for fitting artifacts}

We will try to correct the data for possible artifacts, by using the (systematic) deviations found in Sect. 2.3. Correcting the observed ages based on the absolute numbers deviating from the one-to-one relation is not useful, since the number of clusters that were used as input at each age and mass differs from the observed number. From the input sample we can derive how many clusters are (systematically) fitted with wrong ages and masses. Let the total number of bins in age/mass space be $K$. Here we define the number of bins as the number of bins which are not affected by the detection limit (see Fig. 1, middle panel).
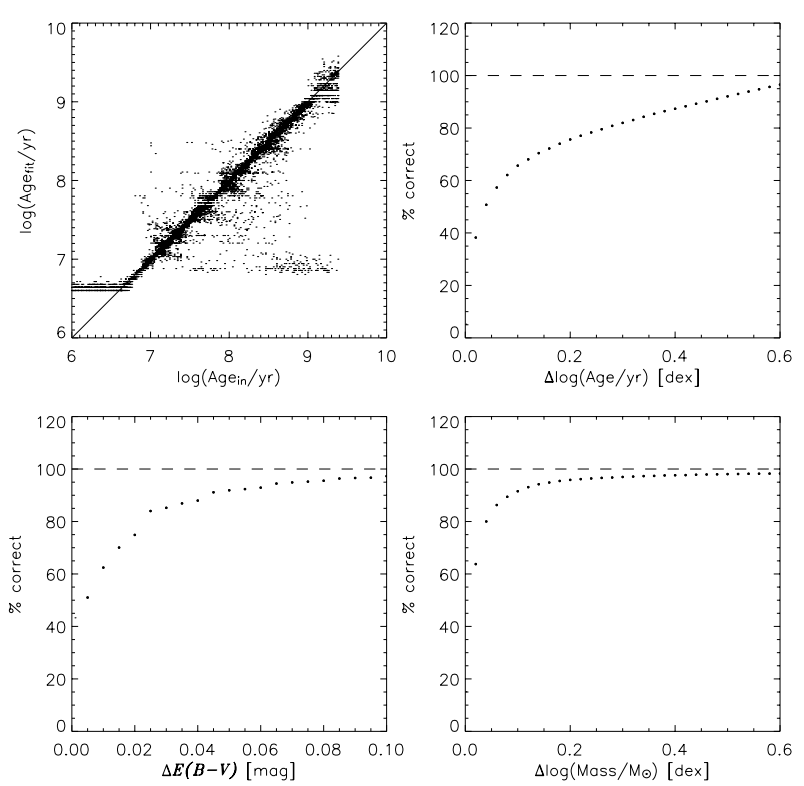

Fig. 3. Result of fitting an artificial cluster sample with the 3DEF method. Top left: the fitted age is shown versus the input age for each cluster. Deviations from the one-to-one relation are caused by photometric errors which are applied to the input sample and misfitted extinction. Top right: the percentage of clusters fitted with the same age as the input value, plus some deviations, as a function of this deviation. Bottom left: similar, but then for extinction. Bottom right: similar but for the mass.

The number of clusters found in each bin is the sum of the contribution of clusters from all bins to this one, where the majority will be from the bin with the same input age and mass. When we write the number of clusters in each bin as a vector with $K$-entries, the fitted number of clusters can be written as a matrix multiplication of all contributions times the input number of clusters

$\left(\begin{array}{c}N_{1} \\ N_{2} \\ \vdots \\ N_{K}\end{array}\right)_{\text {obs }}=\left(\begin{array}{cccc}C_{11} & C_{12} & \ldots & C_{1 K} \\ C_{21} & C_{22} & \ldots & C_{2 K} \\ \vdots & \vdots & \ddots & \vdots \\ C_{K 1} & C_{K 2} & \ldots & C_{K K}\end{array}\right)\left(\begin{array}{c}N_{1} \\ N_{2} \\ \vdots \\ N_{K}\end{array}\right)_{\text {intr }}$

where $N_{\text {intr, }, j}$ is the number of clusters generated in bin $j, N_{\mathrm{obs}, i}$ the number of clusters fitted in bin $i$, and $C_{i j}$ the contribution to bin $i$ from bin $j$. Tests have shown that the best results are acquired when only taking bins into account which are not affected by the detection limit (see Fig. 1, middle panel). From the simulated and fitted sample we can derive the values for $C_{i j}$ for all combinations of $i$ and $j$. All values on the diagonal of $C$ (i.e. $C_{i j}$ where $i=j$ ) are close to 1 , since most clusters are fitted with the same age, extinction, and mass. All other values are 0 or between 0 and 1 . When we know the matrix $C$, the inverse can be used to correct the observations for systematic 3DEF fitting artifacts:

$N_{\text {intr }}=C^{-1} \times N_{\text {obs }}$

where $\boldsymbol{N}_{\text {intr }}$ is the vector with the intrinsic number of clusters, $C^{-1}$ the inverse of the contribution matrix as defined in Eq. (1), and $N_{\text {obs }}$ the vector with observed clusters. When we correct 


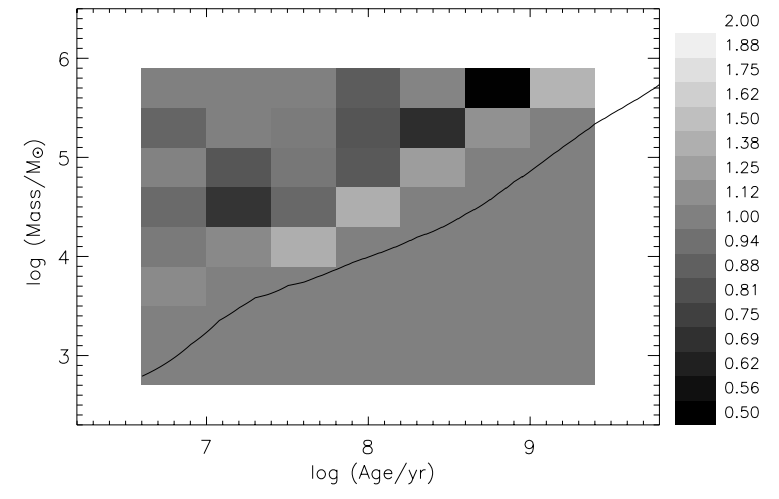

Fig. 4. Ratio of the corrected observations over the uncorrected observations. The corrected data was calculated using Eqs. (1) and (2). Light regions indicate where less clusters are found by the fitting procedure. Dark regions indicate where more clusters are fitted than inputted.

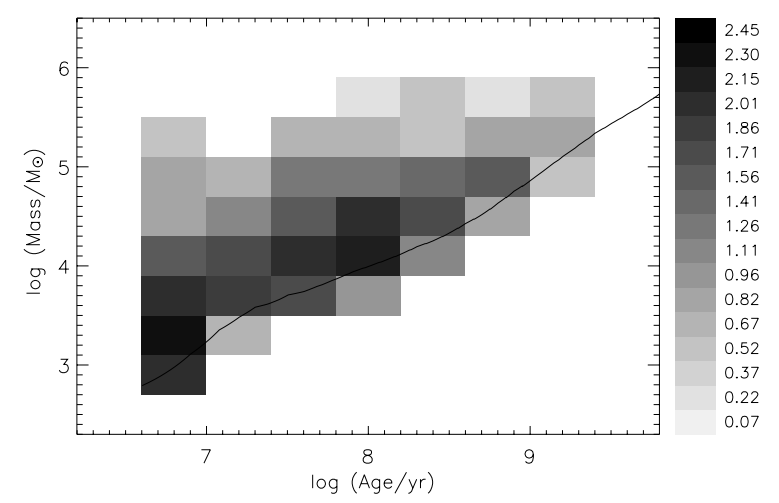

Fig. 5. Corrected age and mass distribution. The raw data from Fig. 1 (bottom) was multiplied with the inverse of the contribution matrix (Eq. (1)).

the observed vector and plot it in a 2D age-mass diagram again, then divide the corrected and uncorrected observations, we can see where deviations take place. Figure 4 shows the ratio of corrected over uncorrected observations. The number of clusters in the age bins at $\log (\mathrm{Age} / \mathrm{yr})=7.2$ and 8.0 has been lowered by about $15 \%$. The observations, however, show a gap at $\log ($ Age $/ y r)=7.2$ (Fig. 1, top), so the underestimation of clusters in that age bin is not caused by our fitting routine.

The corrected observations based on Eq. (2) are shown in Fig. 5. The burst at between 50-70 Myr is less pronounced, but still present. The differences with the uncorrected observations (Fig. 1, bottom) are small, so we conclude that our age-fitting method (3DEF) and our applied selection affect is not severely affecting our age-mass diagrams in a systematic way. In particular, there is no large systematic shift from old to young clusters or the other way around. We therefore conclude that we can use the uncorrected data, as well as the corrected data, to compare with the synthetic cluster populations in Sect. 5. In Sect. 6.2 we show that both the corrected as the uncorrected data give the same results when fitting the analytical models to the data.

\section{Exploration of the parameters which determine the disruption time}

\subsection{Cluster initial mass}

If cluster relaxation drives the evaporation of clusters, then the more massive clusters live longer than their low mass counterparts. Boutloukos \& Lamers (2003) propose an empirical way to determine the dependence of the cluster disruption time on the initial cluster mass, assuming a power-law dependence of the disruption on the cluster mass

$t_{\text {dis }}=t_{4}\left(M_{\mathrm{i}} / 10^{4} M_{\odot}\right)^{\gamma}$

where $t_{4}$ is the disruption time of a $10^{4} M_{\odot}$ cluster, $M_{\mathrm{i}}$ the initial mass of the cluster, and $\gamma$ a dimensionless index. The value of $\gamma$ was determined by measuring the slope of the age and mass distributions of a cluster population. Their mean value for $\gamma$ based on four different galaxies was $\langle\gamma\rangle=0.62 \pm 0.06$.

In a recent study by Lamers et al. (2005a), these observational results are compared with results of $N$-body simulations. The value of $\gamma$ can be explained by tidally driven relaxation and was confirmed to be 0.62 by $N$-body simulations. The explanation for this is that the disruption time of a cluster in a tidal field depends on the relaxation time $\left(t_{\text {rel }}\right)$ and on the crossing time $\left(t_{\mathrm{cr}}\right)$ of the cluster as (Baumgardt 2001):

$t_{\mathrm{dis}} \propto t_{\mathrm{rel}}^{x} \times t_{\mathrm{cr}}^{1-x}$.

Using the expression for $t_{\mathrm{rel}}$ and $t_{\mathrm{cr}}$ from Spitzer (1987), this implies: $t_{\text {dis }} \propto \beta(N / \ln N)^{x}$. Baumgardt \& Makino (2003) found two combinations of $\beta$ and $x$, depending on the concentration of the clusters. Lamers et al. (2005a) showed that for both combinations, $t_{\text {dis }}$ could be well approximated with $t_{\text {dis }} \propto N^{0.62} \propto$ $M^{0.62}$, where $N$ is the number of stars in the clusters and $M$ the total mass of the cluster. King (1958) already had theoretical arguments to expect that the lifetime of clusters should depend on the mass as $t_{\mathrm{dis}} \propto M^{2 / 3}$. The agreement between observations and $N$-body simulations was first noted by Gieles et al. (2004). The physical background of why the disruption time does not scale directly with the relaxation time is given by Fukushige \& Heggie (2000).

\subsection{Cluster radius}

Young clusters are not only affected by the external tidal field of the host galaxy, but they also undergo shocks from (giant) molecular clouds. Both these processes shorten the lifetime of clusters. For both cases, the radius of the cluster is an important parameter in determining how fast the cluster will disrupt. However, both processes depend in very different ways on the radius. From Eq. (4) and the expression for the relaxation time and crossing time, it follows that for the tidally driven relaxation the disruption time depends on the radius as $t_{\mathrm{dis}} \propto r_{\mathrm{h}}^{3 / 2}$. Larger clusters live longer since they have a longer relaxation time, so it takes more time for stars to reach the tidal radius and leave the cluster. Spitzer (1958) has shown that the time needed for a cluster to get unbound due to external shocks relates to the half mass radius of the cluster as $t_{\mathrm{sh}} \propto r_{\mathrm{h}}^{-3}$, so here larger clusters live shorter (for isolated clusters). 


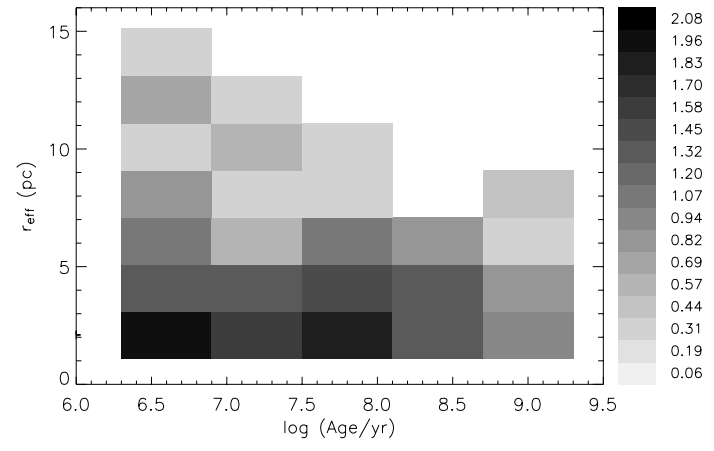

Fig. 6. Number density plot of $r_{\text {eff }}$ vs. age. The decreasing number going towards larger radii shows the power law behavior of the radius distribution. The right hand side shows how the grey values correspond to the logarithm of number of clusters.

To see whether the radius of a cluster is an important parameter in disruption, we used the radii measurements of Paper II. There we measured the projected half light radius (or effective radius) $r_{\text {eff }}$, which relates to the half mass radius as: $r_{\text {eff }}=3 / 4 r_{\mathrm{h}}$ (Spitzer 1987). We made a number density plot of $r_{\text {eff }}$ vs. age for all clusters (Fig. 6). There are clearly no old clusters with large radius, while the opposite is expected due to the size-of-sample effect (Hunter et al. 2003). This suggests that large clusters are disrupted preferentially, which in turn suggests that shocks may be the dominating disruption effect. However, when a large fraction of the clusters is removed, independent of radius, the upper radius also goes down. This is a result from number statistics: less clusters in a power law distribution will result in a lower maximum value. So what really matters here is whether the slope of the radius distribution changes in time or not. In Paper II it is shown that the cluster radius distribution of M 51 is $N(r) \mathrm{d} r \propto r^{-\eta} \mathrm{d} r$, with $\eta=2.2 \pm 0.2$. To see how the slope of the distribution depends on age, we divide our cluster sample in young $(\log (\mathrm{Age} / \mathrm{yr})<7.5)$ and old $(\log ($ Age $/ \mathrm{yr})>7.5)$. Dividing the sample at $\log (\mathrm{Age} / \mathrm{yr})=7.5$ yields two samples of more or less equal size, which gives similar errros in the fit to both distributions. When we determine this index $\eta$ for only young clusters, we find $\eta=2.0 \pm 0.4$, and for old clusters we find $\eta=2.5 \pm 0.6$, which is very similar to the value found for the globular clusters in our Milky Way $(\eta=2.4 \pm 0.2$, Paper II). Although the radius distribution seems to get steeper with age, the errors are too large to place a strong constraint on this. We therefore do not take the radius into account as a free parameter when modeling the cluster disruption. Futher studies of M 51 with higher resolution, for example with the Advanced Camera for Surveys (ACS), could shed light on how the radius of clusters affects the lifetime.

\subsection{Distance to the galactic center}

Lamers et al. (2005a) and Baumgardt \& Makino (2003) have shown that the disruption time is expected to depend on the galactocentric distance of the cluster, the orbital velocity in the galaxy, and the ambient density of the galaxy as

$$
\begin{aligned}
t_{\mathrm{dis}} & \propto R_{\mathrm{G}} / V \\
& \propto \rho_{\mathrm{amb}}^{-0.5}
\end{aligned}
$$

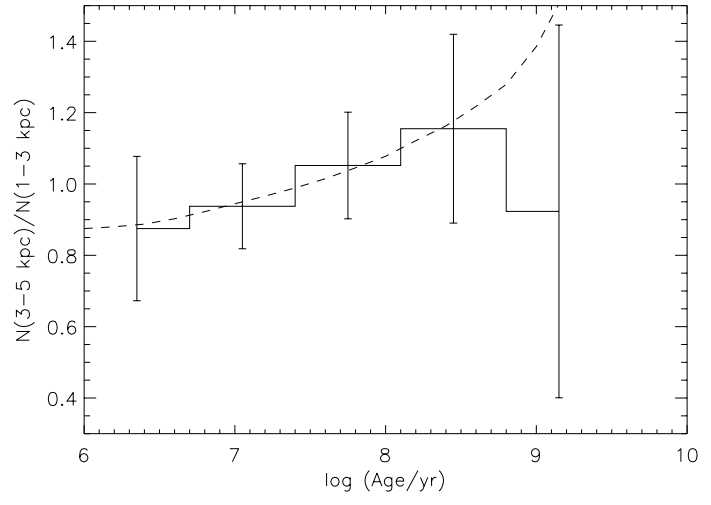

Fig. 7. Ratio of the number of clusters $(N)$ between $3-5 \mathrm{kpc}$ and $1-3 \mathrm{kpc}$ as a function of age. Overplotted is a model predicting this ratio for two disruption times differing a factor of 1.8 , based on Eq. (8).

where $R_{\mathrm{G}}$ is the distance to the galactic center, $V$ the rotational velocity of the cluster in the host galaxy at that distance, and $\rho_{\text {amb }}$ the ambient density of the galaxy at the location of the cluster. The relation with the ambient density holds only when a logarithmic potential is assumed. Since we are dealing with a disk galaxy, it is not so straightforward to derive the ambient density from the spherically symmetric logarithmic potential. Therefore, we prefer the relation with the galactocentric distance and the velocity (Eq. (5)). With Eq. (5) we are able to estimate if we would be able to observe a difference in disruption time at different locations in the galaxy. When we look at clusters between 1 and $3 \mathrm{kpc}$ and at clusters between 3 and $5 \mathrm{kpc}$, the average value of $R_{\mathrm{G}}$ goes up by a factor of 2 . The rotational velocity of M 51 increases from $200 \mathrm{~km} \mathrm{~s}^{-1}$ to $225 \mathrm{~km} \mathrm{~s}^{-1}$ (Rand 1993), so from Eq. (5) we expect the disruption time in the two samples to be different by a factor of 1.8. In Fig. 7 we plot the ratio of the number of clusters at different ages for the outer region $(3-5 \mathrm{kpc})$ and inner region $(1-3 \mathrm{kpc})$. Overplotted is the predicted ratio using Eq. (8) for a disruption timescale that is different by a factor of 1.8. Just as for the radius dependence, we see that the distance to the galactic center plays a role, but the data is not sufficient to include in our analysis.

In conclusion, we see evidence of radius and galactocentric distance-dependent disruption, but the noise is too large to include these parameters in the models. The mass of the cluster is the most dominating parameter in the determination of disruption time, and in the remainder of the study, we only use the mass dependence as a parameter we vary in the models.

\section{Input parameters for modeling the cluster population of M 51}

So far, analytical models for finding the cluster disruption time have assumed that clusters were formed with a constant CFR, as is probably the case for Galactic open clusters (Boutloukos \& Lamers 2003; Battinelli \& Capuzzo-Dolcetta 1991). Lamers et al. (2005b) predicted the age distribution of open clusters. In the case of M 51, we have age and mass information available for each cluster, so predictions can be done for age and mass. In addition, assuming a constant CFR for M 51 
might be an oversimplification of the situation, since the galaxy is in interaction with NGC 5195. In the next sections we explore a broader parameter space.

Since there are strong arguments to believe that the mass dependence of the cluster disruption $(\gamma=0.62)$ is constant (Lamers et al. 2005a), we start by varying only the constant $t_{4}$, to be able to compare our results with clusters gradually losing mass with the instantaneous disruption assumption (Eq. (3)) results of Boutloukos \& Lamers (2003). Next, a two-dimensional parameter search for $\gamma$ and $t_{4}$ is performed, to verify the assumed value for $\gamma$ and to study the dependence of $t_{4}$ on the value of $\gamma$.

Once we have a first estimate of the disruption time, we will study how this value changes when we assume that the CFR has been increasing during the last Gyr or contains bursts at the moments of encounter with NGC 5195.

\section{Analytical model for generating a cluster population}

\subsection{Setting up a synthetic cluster population}

The synthetic cluster populations will be created in a similar way as in Sect. 2.3. This time, however, we want to include realistic input physics, like the cluster IMF and different formation rates, so creating clusters equally spaced in $\log (\mathrm{Age} / \mathrm{yr})$ and $\log \left(M / M_{\odot}\right)$ will not be adequate. When creating clusters with a realistic CIMF, the number of clusters needed to fully sample the CIMF up to $10 \mathrm{Gyr}$ ago is too high. Therefore each cluster was assigned a weight depending on the initial cluster mass and its age $\left(w\left(t, M_{\mathrm{i}}\right)\right)$, proportional to the expected number of clusters formed at each age and mass. The weight is a function of age and mass, scaled such that the youngest, most massive cluster has a weight of 1

$w\left(t, M_{\mathrm{i}}\right)=\left(t / t_{\min }\right) \times(\alpha-1) \times\left(M_{\mathrm{i}} / M_{\max }\right)^{1-\alpha}$

where $w\left(t, M_{\mathrm{i}}\right)$ is the weight assigned to a cluster with age $t$ and mass $M_{\mathrm{i}}, M_{\max }$ the mass of the most massive cluster in the simulation, $t_{\min }$ the age of the youngest cluster in the simulation, and $\alpha$ the slope of the mass function. When $\alpha$ is chosen 2, i.e. $N(M) \sim M^{-2}$, the weight depends on age and mass simply as: $w\left(t, M_{\mathrm{i}}\right) \propto t / M_{\mathrm{i}}$. When the simulated clusters are binned, the weights of the clusters are counted, yielding a realistic $\log (\mathrm{Age} / \mathrm{yr}) \mathrm{vs.} \log \left(M / M_{\odot}\right)$ diagram similar to Fig. 1 (bottom). The advantage of using points spread equally in $\log (\mathrm{Age} / \mathrm{yr})$ and $\log \left(M / M_{\odot}\right)$, with weights assigned, is that the number of points per bin is constant and it is very easy to create a lot of populations with different formation rates, disruption timescales, etc. in a short time.

In our case the clusters are given weights such that, after binning, the CIMF has a slope of $\alpha=2.1$, as found for M 51 (Paper I) and the Galactic open clusters (Battinelli et al. 1994). The weights enable us to model different formation and disruption scenarios (Sects. 6.2-6.4).

\subsection{Including stellar evolution and cluster disruption}

Baumgardt \& Makino (2003) have shown that stellar evolution (SEV) is an important contributor to the dissolution of young clusters, especially for clusters with low concentrations. They also confirm that clusters dissolve with a power-law dependence of their initial mass as $t_{\mathrm{dis}} \sim M_{\mathrm{i}}^{\gamma}$, where $\gamma=0.62$, in agreement with the empirical determination by Boutloukos \& Lamers (2003). In this study instantaneous disruption after the disruption time was assumed as a first approximation and they find that the typical disruption time ( $t_{4}$, see Eq. (3)) varies a lot for different galaxies. In a recent study (Lamers et al. 2005b), it was shown that there is a simple analytical description of the mass of a cluster as a function of time. It takes into account the effect of mass loss due to stellar evolution, based on the mass loss predicted by the GALEV SSP models (Anders et al. 2003; Schulz et al. 2002) and cluster mass loss due to the tidal fields. The mass of the cluster as a function of time can be approximated well by

$M_{\mathrm{p}}(t)=\left(\left(M_{\mathrm{i}} \mu_{\mathrm{sev}}\right)^{\gamma}-\gamma \frac{t}{t_{0}}\right)^{1 / \gamma}$

where $M_{\mathrm{p}}(t)$ is the present mass of the cluster as a function of its age, $M_{\mathrm{i}}$ the initial mass of the cluster, $\mu_{\mathrm{sev}} \equiv M_{\mathrm{p}}(t) / M_{\mathrm{i}}$ is the fraction of remaining mass after mass loss due to stellar evolution has been subtracted, and $t_{0}$ relates to $t_{4}$ as $t_{4}=t_{0} \times$ $10^{4 \gamma}$. The mass as a function of time, according to the analytical formula, agrees perfectly with the predictions following from $N$-body simulations. Lamers et al. (2005b) have also shown that with this analytical model the age distribution of galactic open clusters can be explained very well.

\section{Fitting observed age-mass distribution to predictions}

\subsection{Determining reduced $\chi^{2}$ values from $2 D$ fits}

Artificial cluster samples with realistic input physics (e.g. a CIMF, cluster disruption, bursts, etc.) can now be generated and compared with the observed age and mass number density distribution.

After calculating the analytically generated cluster population, the model was binned into number density plots in the same way as the observed data (see Sect. 2.2) taking the weights into account. In order to compare the simulated (2D) age-mass density plots with the observations, we used the Poisson Probability Law (PPL) introduced by Dolphin \& Kennicutt (2002) for similar purposes

$\mathrm{PPL}=2 \sum_{i=0}^{N} m_{\mathrm{i}}-n_{\mathrm{i}}+n_{\mathrm{i}} \ln \frac{n_{\mathrm{i}}}{m_{\mathrm{i}}}$

where $N$ the number of bins, $m_{\mathrm{i}}$ is the predicted number by the analytical model in bin $i$, and $n_{\mathrm{i}}$ the observed number of clusters in bin $i$. The value of PPL is similar to $\chi^{2}$, in the sense that lower values imply better fits. We always divide the PPL value by the number of bins minus the number of degrees of freedom, which is equivalent to the reduced $\chi^{2}\left(=\chi_{v}^{2}\right)$. We will refer to $\chi_{v}^{2}$ when we discuss results of fits. 


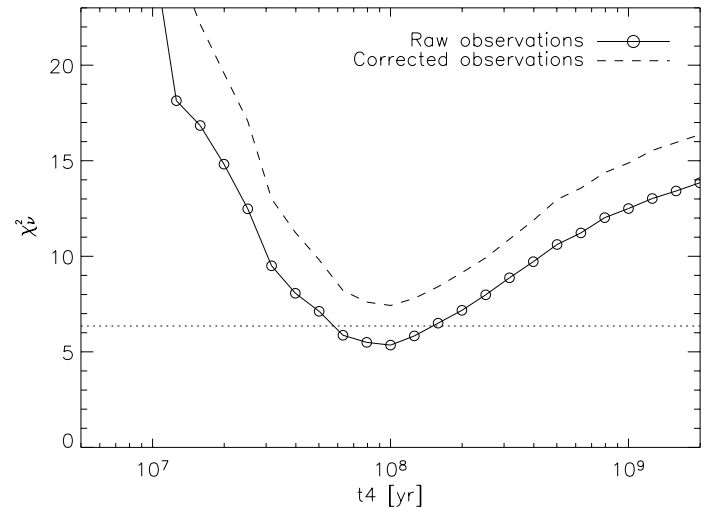

Fig. 8. $\chi_{v}^{2}$ values for different disruption times. A clear minimum is visible at $t_{4}=1.0_{-0.5}^{+0.6} \times 10^{8}$. The dotted line indicates $\chi_{v, \text { min }}^{2}+1$. The dashed line shows a fit to the data after correcting for age-fitting artifacts (Sect. 2.4). In these simulations we have chosen $\gamma$ to be 0.62 , based on theoretical arguments (Sect. 5).

\subsection{Determining the cluster disruption time assuming a constant formation rate of clusters}

To determine the typical cluster disruption time, $t_{4}$, defined in Sect. 5, we generate a cluster sample with a constant CFR and then calculate the cluster masses as a function of age according to Eq. (8) for various values of $t_{4}$. Here we are interested in the disruption time of clusters that have survived that first $10^{7} \mathrm{yr}$ in which the natal cloud is being removed by stellar winds, therefore we excluded the youngest age bin in the fits. Figure 8 shows a clear $\chi_{v}^{2}$ minimum around $t_{4}=1.0_{-0.5}^{+0.6} \times 10^{8} \mathrm{yr}$, where the upper and lower errors are defined by $\chi_{v \text {,accept }}^{-0.5} \leq \chi_{v, \text { min }}^{2}+1$, which is equivalent to the $1 \sigma$ error. In addition, we fitted the same models but then corrected for age-fitting artefacts (Sect. 2.4). The shape of this $\chi_{v}^{2}$ curve is the same as for the raw data, though the values are higher. This shows that the uncertainties of our age-fitting method do not alter the value found for the disruption timescale.

To see how the value of $t_{4}$ depends on the value of $\gamma$, we simulated a grid of cluster populations and varied $t_{4}$ and $\gamma$. A $2 \mathrm{D} \chi_{v}^{2}$ plot is shown in Fig. 9. The minimum is at $\gamma=0.65_{-0.25}^{+0.16}$ and $t_{4}=1.0_{-0.35}^{+0.84} \times 10^{8} \mathrm{yr}$, agreeing very well with the value of $\gamma=0.62$, which was stated earlier based on theoretical arguments and other observational results. The plot also shows that there is a diagonal bar-shaped minimum for different combinations of $t_{4}$ and $\gamma$. One could argue that multiple combinations could be possible, which will yield a somewhat higher value for $t_{4}$. The fit, however, is very sensitive to the choice of bin size when varying two variables. We excluded the mass bins higher than $5 \times 10^{5} M_{\odot}$, since we were probably dealing with a truncation of the mass function. If sampling effects were to determine the upper mass at different ages (Hunter et al. 2003), the maximum mass should increase much more than we observe in the top panel of Fig. 1. This effect makes the mass function steeper above $\log \left(M / M_{\odot}\right) \simeq 5.3$; therefore the region in the age/mass diagram is not suitable to fitting the (sensitive) mass-dependent disruption. An alternative way to measure $\gamma$ would be to measure the slopes of the age and mass distribution separately, as was done in Boutloukos \& Lamers (2003).

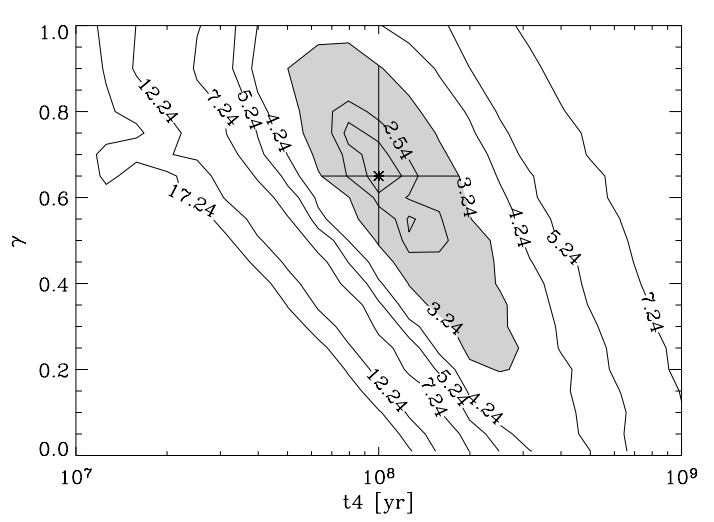

Fig. 9. Two dimensional $\chi_{v}^{2}$ plot of $\gamma$ vs. $t_{4}$. The cross and the shaded area indicate the region where $\chi_{v, \text { min }}^{2}<\chi_{v}^{2}<\chi_{v \text {, min }}^{2}+1$ in $t_{4}$ and $\gamma$.

We fitted these slopes and found the same value for $\gamma$ as for the 2D fit shown in Fig. 9. Again, for the mass, we do not include the high mass end for similar reasons as mentioned before. This method is less sensitive to the choice of bin size, since we can fit the slope of the age and mass distribution independently of the value of the disruption time. We chose to include the result of the simultaneous fit of $t_{4}$ and $\gamma$, because it nicely illustrated how these two variables relate.

\subsection{The effect of an increasing cluster formation rate}

Since NGC 5195 is probably bound to M51 and, therefore, slowly falling in Salo \& Laurikainen (2000), one could argue that the short disruption timescale found in Sect. 6.2 is actually caused by an increasing cluster formation rate (CFR). Bergvall et al. (2003) show that interacting galaxies, such as M 51 (i.e. non-merging), can have an increased star formation rate on the order of a factor of 2-3. We, therefore, model different cluster populations with increasing $\operatorname{CFR}(t)$ rates of various strengths, where we assume that an increasing star formation rate results in an equally large increase in the $\mathrm{CFR}(t)$. We study two different models with increasing CFR: 1.) a linear increasing CFR starting 1 Gyr ago (Sect. 6.3.1) and 2.) a CFR that increases with bursts at the moments of encounter with NGC 5195 (Sect. 6.3.2). Figure 10 gives a schematic illustration of how the CFR varies with time for the two models.

\subsubsection{Linearly increasing cluster formation rate}

In the linear model, the $\operatorname{CFR}(t)$ starts to increase $1 \mathrm{Gyr}$ ago, which is before the moment of the early close encounter with NGC 5195 (400-500 Myr ago, Salo \& Laurikainen 2000). We expect the CFR to start increasing before the moment of the closest encounter, since the two galaxies are already interacting before the first perigalactic passage. We calculated models with different CFR increases and disruption times, and plotted the $\chi_{v}^{2}$ values for various values of $\operatorname{CFR}(t=0) / \operatorname{CFR}\left(t=10^{9} \mathrm{yr}\right)$ and $t_{4}$ in the top panel of Fig. 11.

The minimum $\chi_{v}^{2}$ value is at $t_{4}=2.0_{-1.1}^{+5.2} \times 10^{8} \mathrm{yr}$ and there is an increase in the CFR of 7.0 $0_{-5.0}^{+68.1}$. For low values of $t_{4}$ the equal $\chi_{v}^{2}$ lines are vertical. This can be explained by the fact 


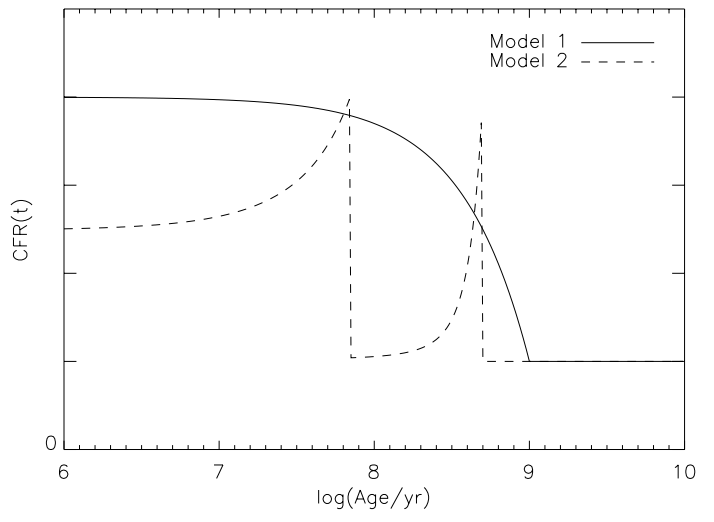

Fig. 10. Illustration of the applied CFR increase in Sect. 6.3 for two different models. Model 1: the CFR is taken to be constant before $1 \mathrm{Gyr}$ ago and then increases linearly in time until $t=0$. Model 2: the CFR increases with steps at the two moments of encounter with NGC 5195. Here the height of the step is the variable.

that, if the disruption time is short, no fingerprints of the ancient formation rate are present in the current population. They are simply erased by disruption. The reason that the equal $\chi_{v}^{2}$ contours are circular around the minimum is that the disruption of clusters depends on the mass of the clusters (Eq. (3)), unlike an increase in the formation rate.

\subsubsection{Cluster formation rate with bursts}

An alternative formation scenario would be that the CFR increases with a burst at the moments of encounter with NGC 5195 and then an exponential decay in the CFR (see model 2 in Fig. 10). We chose the moments of increase at $t=7 \times 10^{7} \mathrm{yr}$ and $t=5 \times 10^{8} \mathrm{yr}$ ago, based on the results of Salo \& Laurikainen (2000), and the typical decay time of the burst is $10^{8} \mathrm{yr}$ (Paper II). The CFR step and $t_{4}$ are varied in different models. The bottom panel of Fig. 11 shows that the lowest $\chi_{v}^{2}$ value is at $t_{4}=2.0_{-1.1}^{+2.3} \times 10^{8} \mathrm{yr}$. This is a factor of 2 higher than when the increasing CFR is not taken into account, but it is the same value as was found for the linear increase in the CFR. The value is still a factor of 5 lower than predicted by $N$-body simulations (Baumgardt \& Makino 2003; Lamers et al. 2005a). The best value for the increase in CFR at the moment of encounter is $3.0_{-1.2}^{+4.6}$. The latter value agrees very well with what is generally observed for the increase in star formation rate of interacting galaxies (Bergvall et al. 2003). Since one of the bursts is clearly observed and a linearly increasing CFR is not so physical, we prefer Model 2 above Model 1. In the next section we compare several properties of this model with the observations.

\subsection{Comparision between the best fit model and the observations}

We show a direct comparison between the age-mass diagrams of the best fit model (Sect. 6.3.2) and the observations in Fig. 12. The densities are scaled such that the total number of simulated clusters equals the total number of observed
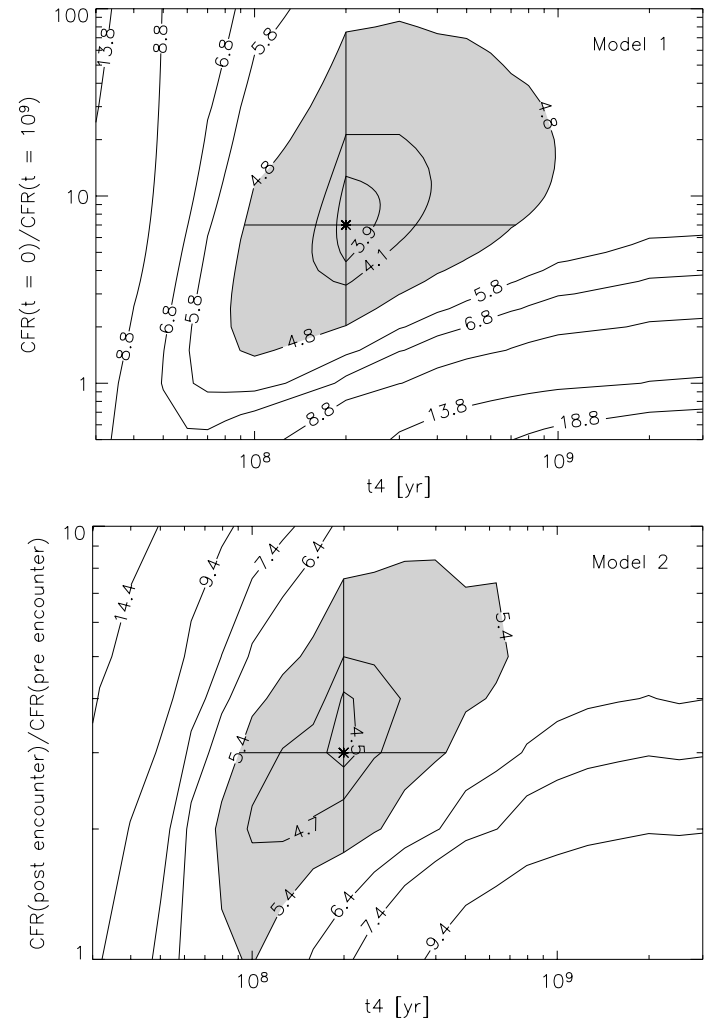

Fig. 11. $\chi_{v}^{2}$ values for different combinations of CFR increase and disruption time $\left(t_{4}\right)$. The top panel shows the results of Model 1 where the CFR increases linearly between $t=1 \mathrm{Gyr}$ and $t=$ present time. The bottom panel shows the result for Model 2, where the CFR increases with steps at the moment of encounter with NGC 5195. The cross and the shaded area indicate the region where $\chi_{v, \text { min }}^{2}<\chi_{v}^{2}<\chi_{v, \text { min }}^{2}+1$.

clusters (1152). A few bins in the observations are empty but not empty in the simulations. The reason for this is that the simulated cluster sample contains bins with values lower than 1 . Apart from this, the general trend of grey values in this $2 \mathrm{D}$ plot is very similar in both cases.

Another interesting property of the observations is the formation rate. In Paper II we showed the number of clusters at different ages for different mass cut-offs. For clusters with masses higher than $10^{4.7} M_{\odot}$, we get a realistic impression of the cluster formation rate. This is because we are complete until $1 \mathrm{Gyr}$ for these masses (see top panel of Fig. 1) and because the most massive clusters are not affected that much by disruption. In Fig. 13 (top) we show the number of clusters in different age bins for the observations and the best fit model. The general trend of the observations is followed very well by the model. A better way to show the formation rate is to divide each age bin by the width of the bin. Then we get the number of clusters formed per unit of time (Myr), as is shown in the bottom panel of Fig. 13. In this figure the over-density of young clusters $(\log (\mathrm{Age} / \mathrm{yr})<7)$ is more obvious and the burst at $7 \times 10^{7} \mathrm{yr}$ is more visible. The first burst of cluster formation $\left(5 \times 10^{8} \mathrm{yr}\right.$ ago $)$ is not visible anymore, since clusters with these ages are already affected by the (short) disruption time. This reinforces that it is very hard to detect variations in the cluster formation rate when the disruption time is that short. 

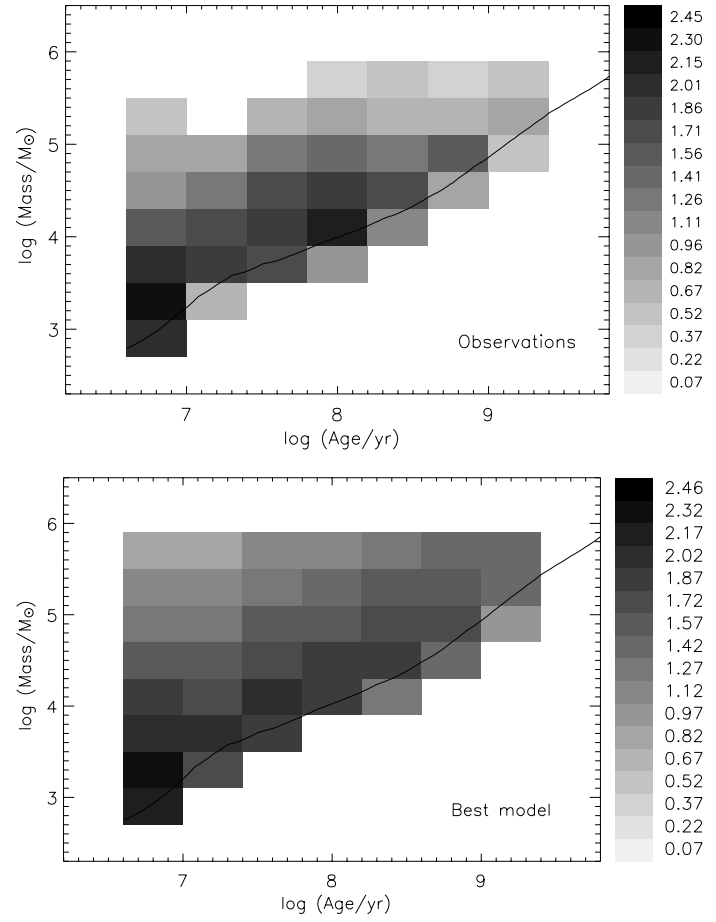

Fig. 12. Comparison between the observed (top) and the modeled (bottom) age vs. mass number density plots. In both plots the $90 \%$ completeness limit of the F439W band is indicated with a line. The right hand side shows how the different grey values correspond to the logarithm of number. The total number in the simulations is scaled to the total number of observed clusters above the $90 \%$ completeness limit (1152).

The largest difference is seen for the bin with $\log (\mathrm{Age} / \mathrm{yr})=7$ and 8.25. The model predicts more clusters in these bins than are observed. This can be explained by fitting artefacts which yield an (unphysical) underdensity of clusters (Sect. 2.3). The model is still within the $3 \sigma$ error of the observations, however.

\section{Implication of the derived disruption time}

We have shown that the clusters disruption time for a typical cluster with mass of $10^{4} M_{\odot}$ is around $10^{8} \mathrm{yr}$ in M 51 . When increasing formation rates are taken into account the disruption time increases by a factor of 2 . This is significantly longer than Boutloukos \& Lamers (2003) find for clusters in a smaller region of M $51\left(t_{4}=4 \times 10^{7} \mathrm{yr}\right)$. This could be because they did not separate the dissolution due to infant mortality rate from the evaporation by the tidal field from the galaxy. Clusters with ages younger than $10^{7} \mathrm{yr}$ are not taken into account in this study, since they are affected by dissolution due to the removal of primordial gas. Theoretical predictions show that clusters of $10^{4} M_{\odot}$ in a tidal field of the strength of M 51 should have a disruption time of about $10^{9} \mathrm{yr}$ (Lamers et al. 2005a; Baumgardt \& Makino 2003). This value is found from observations of clusters in the solar neighborhood (Boutloukus \& Lamers 2003). What causes the clusters in M 51 to dissolve about 5 times faster than predicted? A few effects that have not
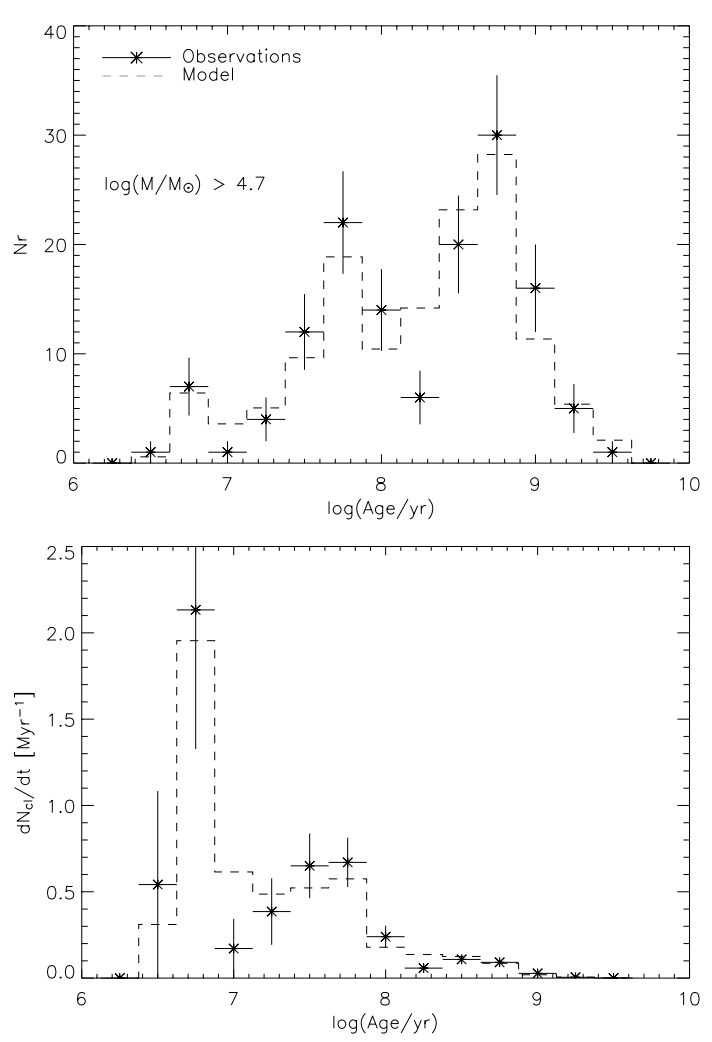

Fig. 13. Comparison of the age distribution of clusters with masses larger than $10^{4.7} M_{\odot}$ and the one derived from the best fit model. Top: number of clusters per age bin. Bottom: the cluster formation rate (number per Myr).

been incorporated in the $N$-body models that predict the disruption times in tidal fields are:

1. Variations in the stellar IMF. When clusters are formed with a so-called top heavy IMF as is observed in the starburst galaxy M 82 (Smith \& Gallagher 2001), clusters will disperse much faster, since the disruption time depends on the number of stars in the clusters as $t_{\text {dis }} \propto N^{0.62}$ (Sect. 3.1). Suppose the stellar IMF starts at $1 M_{\odot}$ instead of $0.1 M_{\odot}$, then the number of stars for a given cluster mass will be about a factor 10 lower. This will make the disruption time a factor $10^{0.62} \simeq 4$ lower, which would nicely explain the factor 5 difference in disruption time we observe.

2. External perturbations. The $N$-body models of Baumgardt \& Makino (2003) calculated the disruption time of clusters in a smooth external potential from the host galaxy. In reality, the cluster will also experience additional external perturbations, for example, the encounters with molecular clouds. The clusters in our sample are in the inner $5 \mathrm{kpc}$ of the galaxy, where most of the giant molecular clouds reside (Henry et al. 2003; Kuno et al. 1995). The encounters with molecular clouds can speed up the disruption of clouds significantly (e.g. Terlevich 1987; Theuns 1991).

3. Out-of-equilibrium formation of clusters. All clusters in the $N$-body models start in virial equilibrium and in tidal equilibrium with the host galaxy. Kroupa (2004) has shown that after the gas removal phase, the clusters are not in virial 
equilibrium anymore, and the outer parts of the cluster have expanded. It will be easier to dissolve these clusters then when all stars are in tidal equilibrium and within the tidal radius imposed by the host galaxy.

4. Variations in the central concentration. The $N$-body models of Baumgardt \& Makino (2003) start clusters with concentration values of $W_{0}=5-7$. This is the average concentration of globular clusters in our Milky Way (Harris 1996). When clusters start with much lower concentration, the core of the clusters is less compact and the cluster will be more vulnerable external perturbations. The concentration of clusters in M 51 cannot be determined due to lack of resolution, but we know from young open clusters in the Milky Way that they have much smaller concentration indices than the globular clusters (Binney \& Tremaine 1987).

So far, the clusters in M 51 have not been checked for variations in the IMF in the way it was done for clusters in other galaxies (e.g. Smith \& Gallagher 2001; Larsen et al. 2004; Maraston et al. 2004). Also, no $N$-body experiments have been performed including the effect of a tidal field and perturbations by giant molecular clouds. Arguments 3 and 4 are based upon unknown observables of young clusters and they could hold for clusters in other galaxies as well.

When the disruption of clusters is indeed as short as we derived, young massive clusters $\left(M_{\mathrm{i}} \simeq 10^{6} M_{\odot}\right)$ will not survive longer than $3.5 \times 10^{9} \mathrm{yr}$. This means that the disk of M 51 is not the right location for young globular clusters to survive over a Hubble time.

\section{Conclusions}

We have compared the cluster population of M 51 with theoretical predictions including evolutionary mass loss, cluster disruption, variable cluster formation rate, and a magnitude limit. The age vs. mass diagrams of the observed cluster populations were binned to acquire two dimensional number density plots, which can be compared with simulated cluster samples. The results can be summarized as follows:

1. Artifacts introduced by our age-fitting routine do not systematically bias our sample towards either young or old clusters. We present a method to correct observations of a cluster population for artifacts introduced by the age-fitting method applied.

2. The size of the largest cluster decreases with age, from $15 \mathrm{pc}$ for clusters with $\log (\mathrm{Age} / \mathrm{yr})<7$ to $10 \mathrm{pc}$ for clusters with ages around $1 \mathrm{Gyr}$. In addition, the slope of the radius distribution seems to get steeper in time: $\eta=2.0 \pm 0.4$ for clusters younger than $\log (\mathrm{Age} / \mathrm{yr})=7.5$ and $\eta=2.5 \pm 0.6$ for clusters with $\log ($ Age $/ y r)>7.5$. Both these results seem to suggest that smaller clusters have a larger chance of surviving. However, the radius distribution of globular clusters in our Milky Way is very similar to the values found: $\eta=2.4 \pm 0.2$. Samples with higher spatial resolution and more clusters are needed to study the radius dependence.

3. There are more old clusters at larger distances from the galactic center. The ratio of the number of clusters in the outer parts of the galaxy $(3-5 \mathrm{kpc})$ over the number of clusters in the inner part (1-3 kpc) per age bin increases with a factor of 1.8 in age (from $\log (\mathrm{Age} / \mathrm{yr})=6.5$ to $\log ($ Age $/ y r)=8.5)$, which is to be expected since the disruption time depends on the distance to the galactic center.

4. Assuming that the cluster disruption time depends on the initial mass of the cluster as $t_{\mathrm{dis}} \propto M_{\mathrm{i}}^{\gamma}$, and using $\gamma=$ 0.62 based on theoretical and observational studies (Lamers et al. 2005a), we find a typical disruption for a $10^{4} M_{\odot}$ cluster of $t_{4}=1.0_{-0.5}^{+0.6} \times 10^{8} \mathrm{yr}$, where we assumed a constant cluster formation rate.

5. When $\gamma$ and $t_{4}$ are varied together, the value found for $\gamma$ is similar to that predicted by Lamers et al. (2005a), based on observational and $N$-body studies. A value of $\gamma=0.65_{-0.25}^{+0.16}$ and $t_{4}=1.0_{-0.35}^{+0.84} \times 10^{8} \mathrm{yr}$ are the best combination.

6. We studied the degeneracy between formation increase and disruption. Models where the cluster formation rate increases linearly in time do not affect the disruption timescale much ( $t_{4}$ gets a factor 2 higher). When we include bursts at the moments of encounter with NGC 5195, the typical disruption time is also a factor of 2 higher.

7. When clusters of $10^{4} M_{\odot}$ are disrupted within $2 \times 10^{8} \mathrm{yr}$, and considering the power-law dependence of the disruption time scale with the initial cluster mass, even clusters with a mass of $10^{6} M_{\odot}$ will not survive longer than $3.5 \mathrm{Gyr}$. This means that the disk of M 51 is not a preferred location to form a new generation of globular clusters. This might explain why so far there are no old ( $>\mathrm{Gyr}$ ) massive $\left(>10^{6} M_{\odot}\right)$ clusters known in the disks of spiral galaxies, although they are still forming: e.g. Westerlund 1 in the Galactic disk (Clark \& Negueruela 2004) and the young globular cluster in NGC 6946 (Larsen et al. 2001).

Acknowledgements. MG acknowledges the support by NOVA-grant 10.10.1.11 to HJGLML.

\section{References}

Anders, P., \& Fritze-v. Alvensleben, U. 2003, A\&A, 401, 1063

Anders, P., Bissantz, N., Fritze-v. Alvensleben, U., \& de Grijs, R. 2004, MNRAS, 347, 196

Bastian, N., \& Lamers, H. J. G. L. M. 2003, in Extragalactic Globular Cluster Systems, ed. M. Kissler-Patig, ESO Astrophysics Symposia (Springer: Berlin), 28

Bastian, N., Gieles, M., Lamers, H. J. G. L. M., Scheepmaker, R. A., \& de Grijs, R. 2005, A\&A, 431, 905 (Paper II)

Battinelli, P., Brandimarti, A., \& Capuzzo-Dolcetta, R. 1994, A\&AS, 104,379

Battinelli, P., \& Capuzzo-Dolcetta, R. 1991, MNRAS, 249, 76

Baumgardt, H. 2001, MNRAS, 325, 1323

Baumgardt, H., \& Makino, J. 2003, MNRAS, 340, 227

Binney, J., \& Tremaine, S. 1987 (Princeton, NJ: Princeton University Press), 747

Bergvall, N., Laurikainen, E., \& Aalto, S. 2003, A\&A, 405, 31

Bik, A., Lamers, H. J. G. L. M., Bastian, N., Panagia, N., \& Romaniello, M. 2003, A\&A, 397, 473 (Paper I)

Boutloukos, S. G., \& Lamers, H. J. G. L. M. 2003, MNRAS, 338, 717

Brodie, J. P., Schroder, L. L., Huchra, J. P., et al. 1998, AJ, 116, 691

Clark, J. S., \& Negueruela, I. 2004, A\&A, 413, 15 
de Grijs, R., Anders, P., Lamers, H. J. G. L. M., et al. 2004, MNRAS, accepted

Dolphin, A. E., \& Kennicutt, R. C. 2002, AJ, 124, 158

Fukushige, T., \& Heggie, D. C. 2000, MNRAS, 318, 753

Geyer, M. P., \& Burkert, A. 2001, MNRAS, 323, 988

Gieles, M., Baumgardt, H., Bastian, N., \& Lamers, H. J. G. L. M. 2004, The formation and evolution of massive young star clusters, ed. H. L. G. L. M. Lamers, L. J. Smith, \& A. Nota (San Francisco: ASP), ASP Conf. Ser., 322, 481

Gieles, M., Larsen, S. S., Bastian, N., \& Stein, I. T. 2005, A\&A, submitted

Harris, W. E. 1996, AJ, 112, 1487

Henry, A. L., Quillen, A. C., \& Gutermuth, R. 2003, AJ, 126, 283

Hodge, P. 1987, PASP, 99, 724

Hunter, D. A., Elmegreen, B. G., Dupuy, T. J., \& Mortonson, M. 2003, AJ, 126, 1836

King, I. 1958, AJ, 63, 306

Kroupa, P. 2004 [arXiv: astro-ph/0412069]

Kuno, N., Nakai, N., Handa, T., \& Sofue, Y. 1995, PASJ, 47, 745

Lada, C. J., \& Lada, E. A. 2003, ARA\&A, 41, 57

Lamers, H. J. G. L. M., Gieles, M., \& Portegies Zwart, S. F. 2005a, A\&A, 429, 173

Lamers, H. J. G. L. M., Gieles, M., Bastian, N., et al. 2005b, A\&A, 441, 117

Larsen, S. S., Brodie, J. P., Efremov, Y. N., et al. 2001, ApJ, 556, 801

Larsen, S. S., Brodie, J. P., \& Hunter, D. A. 2004, AJ, 128, 2295

Maraston, C., Bastian, N., Saglia, R. P., et al. 2004, A\&A, 416, 467

Rand, R. J. 1993, ApJ, 410, 68

Salo, H., \& Laurikainen, E. 2000, MNRAS, 319, 377

Schulz, J., Fritze-v. Alvensleben, U., \& Fricke, K. J. 2002, A\&A, 392, 1

Smith, L. J., \& Gallagher, J. S. 2001, MNRAS, 326, 1027

Spitzer, L. J. 1958, ApJ, 127, 17

Spitzer, L. 1987 (Princeton, NJ: Princeton University Press), 40

Terlevich, E. 1987, MNRAS, 224, 193

Theuns, T. 1991, Mem. Soc. Astron. It., 62, 909 